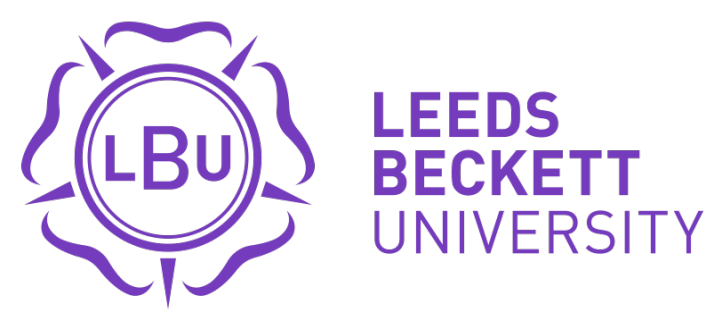

Citation:

Sun, G and Chang, V and Guan, S and Ramachandran, M and Li, J and Liao, D (2018) Big Data and Internet of Things-Fusion for different services and its impacts. Future Generation Computer Systems, 86. pp. 1368-1370. ISSN 0167-739X DOI: https://doi.org/10.1016/j.future.2018.05.022

Link to Leeds Beckett Repository record:

https://eprints.leedsbeckett.ac.uk/id/eprint/7407/

Document Version:

Article

Creative Commons: Attribution-Noncommercial-No Derivative Works 4.0

The aim of the Leeds Beckett Repository is to provide open access to our research, as required by funder policies and permitted by publishers and copyright law.

The Leeds Beckett repository holds a wide range of publications, each of which has been checked for copyright and the relevant embargo period has been applied by the Research Services team.

We operate on a standard take-down policy. If you are the author or publisher of an output and you would like it removed from the repository, please contact us and we will investigate on a case-by-case basis.

Each thesis in the repository has been cleared where necessary by the author for third party copyright. If you would like a thesis to be removed from the repository or believe there is an issue with copyright, please contact us on openaccess@leedsbeckett.ac.uk and we will investigate on a case-by-case basis. 


\title{
Editorial: Big Data and Internet of Things - Fusion for different services and its impacts
}

\author{
Gang Sun ${ }^{a, b}$, Victor Chang c, d, Steven Guan ${ }^{\text {d, e }}$, Muthu Ramachandran ${ }^{f}$, Jin Li ${ }^{g}$, Dan Liao ${ }^{a, b}$
}

a. Key Lab of Optical Fiber Sensing and Communications (Ministry of Education), UESTC, Chengdu, 611731, China

b. Guangdong Institute of Electronic and Information Engineering, UESTC, 523808, China

c. International Business School Suzhou, Xi'an Jiaotong-Liverpool University, Suzhou, China

d. Research Institute of Big Data Analytics, Xi'an Jiaotong-Liverpool University, Suzhou, China

e. School of Computer Science and Software Engineering, Xi'an Jiaotong-Liverpool University, Suzhou, China

f. School of Computing, Creative Technologies and Engineering, Leeds Beckett University, UK

g. School of Computer Science, Guangzhou University, Guangzhou, China

\section{Introduction}

Cloud computing emerged to integrate computing resources and increase the efficiency and cost of the technologies for businesses. The emergence of Big Data and loT have provided new dimensions and opportunities for improved analytics and predictions [1]. This motivates us to develop a special issue on the fusion between Big Data and IoT. The fusion between Big Data and loT can create impacts in the future generation of our development in smart cities, national planning and forecasting of our future activities and investments. Big Data and IoT fusion also supports directly on pervasive technology to our daily life in healthcare, finance, security, transportation and education [2-6]. To enable future generation of different services, we need to understand and realize the significance of fusion between hardware and software, and between security and reality. By doing so, we can get very light and portable devices that can contain petabytes of data, which need layers of security functions and services to make them protected. We can also use one device that can be a mobile phone, instant messenger, video conferencing center, GPS, database, investment analytics, weather forecaster, camera and data processing center. We can also provide real time security services that can destroy a vast variety of Trojans and viruses, block all security breaches, restore things back to normal and keep the owners alert and safe in real time. Big Data and loT fusion can help high-tech sectors such as weather forecasting, space technology and biotechnology to enable thousands of simulations to be completed in seconds $[1,4,6-10]$. All these high tech features have become reality and not just in movies enabled by the impacts of Big Data and loT fusion.

We received 34 submissions altogether. After conducting a very vigorous review process and a high selection criteria by independent reviewers, only six papers were selected. We have achieved $17.64 \%$ acceptance rate to reflect the high expectations and academic merits on all the papers. 


\section{Our selected papers}

This special issue presents interesting, pioneering and innovative articles on research topics related to the fusion of big data and loT based applications:

\section{Video big data in the smart city}

The paper on video big data in the smart city: Background construction and optimization for surveillance video processing by L. Tian et al. [11] proposes a three level video data fusion scheme, a coding based architecture proposed for smart city video, and a specific coding parameter optimisation algorithm is also been proposed to measure performance attributes of the proposed applications. The proposed approach will enhance smart city applications, in particular, sensing, monitoring, and analysing video based data from transforming infrastructures, building, and transports to achieve efficient smart cities. In addition, the proposed algorithms on a block-level background modeling (BBM) supporting long-term reference structure for efficient surveillance video coding, the ratedistortion optimization for surveillance source (SRDO) algorithm is also developed to improve the coding performance. Experimental results show that the proposed BBM and SRDO can significantly improve the compression performance, which can effectively support diverse video applications in smart city.

\section{A hybrid model of Internet of Things and cloud computing}

The article on a hybrid model of Internet of Things and cloud computing to manage big data in health services applications by M. Elhoseny et al. [12] has proposed an interesting approach to big data in health services with their approach to optimise virtual machines. One of the interesting approaches proposed in this paper is a new model to optimize virtual machines selection (VMs) in cloud-loT health services applications to efficiently manage a big amount of data in integrated industry 4.0. Industry 4.0 applications has evolved that require to process and analyze big data, which come from different sources such as sensor data, without human intervention. The proposed model aims to enhance the performance of the healthcare systems by reducing the stakeholders' request execution time, optimizing the required storage of patients' big data and providing a real-time data retrieval mechanism for those applications. The architecture of the proposed hybrid cloud-loT consists of four main components: stakeholders' devices, stakeholders' requests (tasks), cloud broker and network administrator. This paper has made a novel contribution to optimize the VMs selection with three different well-known optimizers (Genetic Algorithm (GA), Particle swarm optimizer (PSO) and Parallel Particle swarm optimization (PPSO) are used to build the proposed model.

\section{Clustering big loT data}

The article on clustering big loT data by metaheuristic optimized mini-batch and parallel partitionbased DGC in Hadoop by R. Tang and S. Fong [13] have proposed a parallel computing algorithm based on k-means approach to performance improvement. To this end, this paper proposes a new partitioned clustering method that is optimized by metaheuristic for loT big data environment. The proposed method has three main activities such as a sample of the dataset is partitioned into mini batches, followed by adjusting the centroids of the mini batches of data, and is collating the mini batches to form clusters, so the quality of the clusters would be maximized. They also define the notion of Dynamic Group Optimisation which controls the positions of the centroids that could be optimally attuned at the mini batches and are governed by a metaheuristic. The results show that our proposed method is a promising tool for clustering fused loT data efficiently. 


\section{Multimedia Internet of Things}

The paper on improving quality of experience in multimedia Internet of Things leveraging machine learning on big data by Xiaohong Huang et al. [14] has studied the problem of automatic optimization of Quality of Experience (QOE) through collecting and processing various data from multimedia loT (MloT) networks and designed a QoE optimization mechanism for MloT leveraging data fusion technology, which consists of two steps: i) a multimodal data fusion approach is proposed to build a QoE mapping between the uncontrollable user data with the controllable network-related system data; and ii) an automatic QoE optimization model is built taking fused results, which is different from the traditional way. The proposed mechanism is able to adjust network-related system data automatically and thus achieve optimized user satisfaction. The results show that the proposed mechanism can improve the QoE level significantly as well as be adaptable to dynamic network changes.

\section{Security Fusion as a Service}

The article on SFaaS: Keeping an eye on loT fusion environment with security fusion as a service by Chien-Ting Kuo et al. [15] has discussed the security vulnerability threat and zero-day attacks that could happen in the SDN fusion environment if the switches are compromised through vulnerabilities and addressed the SDN security challenge of compromised switches. The authors proposed a concept of Security Fusion as a Service (SFaaS) including two detection mechanisms for detecting the incorrect forwarding, duplicated forwarding, and malicious weight adjusting in the preliminary research. For solving the studied issue and creating a more flexible detecting service of SFaaS, they developed a softwarized switch topology measurement architecture for developing $100 \%$ reliable testing switches for the detection environment. The simulation results show that the proposed architecture and detecting mechanism are useful for reducing damage and thus to prove the validity of the proposed concept.

\section{Privacy-preserving fusion of loT and big data}

The paper on privacy-preserving fusion of loT and big data for e-health by Yang Yang et al [16]. has investigated the privacy-preserving fusion of IoT and big data in the e-health applications, and constructed a system to realize secure loT communication and confidential medical big data storage. The medical big data contains a large amount of electronic health records (EHRs) are outsourced to cloud platform. In the proposed system, the patient distributes an loT group key to the medical nodes in an authenticated way without interaction round. Usually, the loT messages are encrypted using the IoT group key and transmitted to the patient, which can be batch authenticated. The encrypted EHRs are shared among patient and different data users in a fine-grained access control manner. The authors have designed a novel keyword match based policy update mechanism to enable flexible access policy updating without privacy leakage. The results demonstrated that the proposed system and algorithms are efficient.

\section{Conclusion}

We thank FGCS and Prof Peter Sloot very much for providing us the opportunities to serve the community. We have received numerous words of support, encouragement and constructive feedback. We are honored to publicize this special issue. We are certain that more institutions and organizations will use fusion in various aspects, such as service fusion, data fusion, fusion in Industry 4.0 etc. which can blend with big data, IoT, security and cloud computing effectively. Our selected 
papers and related work will hope to make greater impacts and contributions to different scientific communities. We also look forward to meeting our honorable Prof Peter Sloot in Wuxi, China on $11^{\text {th }}$ June, 2018 and the possibility of having the long-term collaboration.

\section{References}

[1] Gang Sun, Victor Chang, Guanghua Yang, and Dan Liao. The cost-efficient deployment of replica servers in virtual content distribution networks for data fusion. Information Sciences (2018), 432, 495515.

[2] Jayavardhana Gubbi, Rajkumar Buyya, Slaven Marusic, and Marimuthu Palaniswami. Internet of Things (IoT): A vision, architectural elements, and future directions. Future generation computer systems 29, no. 7 (2013): 1645-1660.

[3] Seungwoo Jeon, Bonghee Hong, and Victor Chang. Pattern graph tracking-based stock price prediction using big data." Future Generation Computer Systems 80 (2018): 171-187.

[4] Jing Qi, Athanasios V. Vasilakos, Jiafu Wan, Jingwei Lu, and Dechao Qiu. Security of the Internet of Things: perspectives and challenges. Wireless Networks 20, no. 8 (2014): 2481-2501.

[5] Yang Yang, Xianghan Zheng, Wenzhong Guo, Ximeng Liu, and Victor Chang. Privacy-preserving smart loT-based healthcare big data storage and self-adaptive access control system. Information Sciences (2018).

[6] Bossé Éloi, and Basel Solaiman. Information fusion and analytics for big data and loT. Artech House, 2016.

[7] Hongming Cai, Boyi Xu, Lihong Jiang, and Athanasios V. Vasilakos. loT-based big data storage systems in cloud computing: perspectives and challenges. IEEE Internet of Things Journal 4, no. 1 (2017): 75-87.

[8] Qingchen Zhang, Laurence T. Yang, Zhikui Chen, and Peng Li. High-order possibilistic c-means algorithms based on tensor decompositions for big data in loT. Information Fusion 39 (2018): 72-80.

[9] Sowe Sulayman K., Takashi Kimata, Mianxiong Dong, and Koji Zettsu. Managing heterogeneous sensor data on a big data platform: IoT services for data-intensive science. In Computer Software and Applications Conference Workshops (COMPSACW), 2014 IEEE 38th International, pp. 295-300. IEEE, 2014.

[10] Victor Chang. Towards data analysis for weather cloud computing. Knowledge-Based Systems 127 (2017): 29-45.

[11] Ling Tian, Hongyu Wang, Yimin Zhou, and Chengzong Peng. Video big data in smart city: Background construction and optimization for surveillance video processing. Future Generation Computer Systems (2018).

[12] Mohamed Elhoseny, Ahmed Abdelaziz, Ahmed S. Salama, A. M. Riad, Khan Muhammad, and Arun Kumar Sangaiah. A hybrid model of Internet of Things and cloud computing to manage big data in health services applications. Future Generation Computer Systems (2018).

[13] Rui Tang, and Simon Fong. Clustering big loT data by metaheuristic optimized mini-batch and parallel partition-based DGC in Hadoop. Future Generation Computer System (2018). 
[14] Huang, Xiaohong, Kun Xie, Supeng Leng, Tingting Yuan, and Maode Ma. Improving quality of experience in multimedia Internet of Things leveraging machine learning on big data. Future Generation Computer Systems (2018).

[15] Chien-Ting Kuo, Po-Wen Chi, Victor Chang, and Chin-Laung Lei. SFaaS: Keeping an eye on loT fusion environment with security fusion as a service. Future Generation Computer Systems (2018).

[16] Yang, Yang, Xianghan Zheng, Wenzhong Guo, Ximeng Liu, and Victor Chang. Privacy-preserving fusion of IoT and big data for e-health. Future Generation Computer Systems (2018).

\section{Biography}

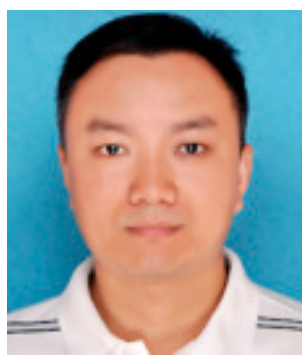

Gang Sun is an associate professor of Computer Science at University of Electronic Science and Technology of China (UESTC). His research interests include network virtualization, cloud computing, high performance computing, parallel and distributed systems, ubiquitous/pervasive computing and intelligence and cyber security. He has co-authored 80 technical publications including paper in refereed journals and conferences, invited papers and presentations and book chapters. He has been a visiting fellow at The Australian National University (2015-2016). He is a Member of IEEE/IEEE Computer Society. He has also edited special issues at top journals, such as Future Generation Computer Systems and Multimedia Tool and Applications. He has served as reviewers of IEEE Transactions on Industrial Informatics, IEEE Communications Letters, Information Fusion, Future Generation Computer Systems, Journal of Network and Computer Applications, Journal of Supercomputing, Journal of Parallel and Distributed Computing, KSII Transactions on Internet and Information Systems, Computers and Electrical Engineering, Chinese Journal of Electronics.

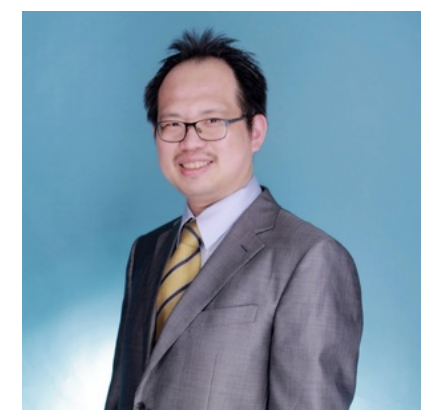

Victor Chang is an Associate Professor (Reader), Director of PhD and Director of MRes at IBSS, Xi'an Jiaotong-Liverpool University (XJTLU), Suzhou, China, since June 2016. He is also a very active and contributive key member at Research Institute of Big Data Analytics (RIBDA), XJTLU. Previously he worked as a Senior Lecturer at Leeds Beckett University, UK, for 3.5 years. Within 4 years, he completed Ph.D. (CS, Southampton) and PGCert (Higher Education, Fellow, Greenwich) while working for several projects at the same time. Before becoming an academic, he has achieved $97 \%$ on average 
in 27 IT certifications. He won a European Award on Cloud Migration in 2011, IEEE Outstanding Service Award in 2015, best papers in 2012 and 2015, the 2016 European award: Best Project in Research, 2016 SEID Excellent Scholar, Suzhou, China, Outstanding Young Scientist award in 2017, 2017 special award on Data Science, 2017 and 2018 INSTICC Service Awards and numerous awards since 2012. He is a visiting scholar/PhD examiner at several universities, an Editor-in-Chief of IJOCI \& OJBD journals, Editor of FGCS, Associate Editor of TII, founding chair of two international workshops and founding Conference Chair of loTBDS http://www.iotbd.org and COMPLEXIS http://www.complexis.org since Year 2016. He was involved in different projects worth more than $f 12.5$ million in Europe and Asia. He has published 3 books as sole authors and the editor of 2 books on Cloud Computing and related technologies. He gave 16 keynotes at international conferences. He is widely regarded as one of the most active and influential young scientist and expert in IoT/Data Science/Cloud/security/AI/IS.

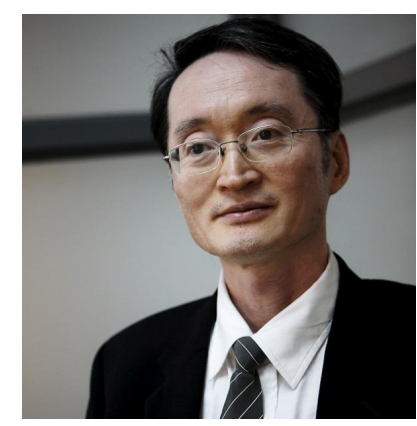

Steven Guan received his M.Sc. \& Ph.D. from the University of North Carolina at Chapel Hill. He is currently a Professor and the Director for Research Institute of Big Data Analytics at Xi'an JiaotongLiverpool University (XJTLU). He served the head of department position at XJTLU for 4.5 years, creating the department from scratch and now in shape. Before joining XJTLU, he was a tenured professor and chair in intelligent systems at Brunel University, UK. Prof. Guan has worked in a prestigious R\&D organization for several years, serving as a design engineer, project leader, and department manager. After leaving the industry, he joined Yuan-Ze University in Taiwan for three and half years. He served as deputy director for the Computing Center and the chairman for the Department of Information \& Communication Technology. Later he joined the Electrical \& Computer Engineering Department at National University of Singapore as an associate professor. Prof. Guan's research interests include: machine learning, data mining, data analytics, modeling, security, networking, mobile commerce, coding theory, and pseudorandom number generation. He has published extensively in these areas, with $130+$ journal papers and $180+$ book chapters or conference papers. He has chaired and delivered keynote speeches for 30+ international conferences and served in $170+$ international conference committees and $20+$ editorial boards.

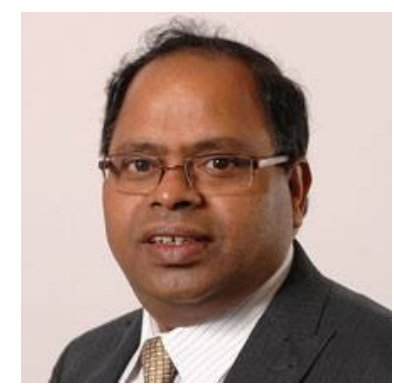


Muthu Ramachandran Dr Muthu Ramachandran is currently a Principal Lecturer (Associate Professor) in the School of Computing, Creative Technologies, and Engineering at Leeds Beckett University in the UK. Muthu has extensive research experience coupled with a teaching background and experiences on Cloud Software Engineering, Big Data Software Engineering, software and systems engineering methods \& lifecycle, software development, agile software engineering, project management skills, process improvement skills, internet technology, mobile, networks, and distributed computing, realtime \& embedded systems, cloud computing, service-oriented architecture, and IT systems development for the past 25 years. His first career started as a research scientist at India Space Research Labs where he worked on real-time systems development projects. Muthu is an author of two books: Software Components: Guidelines and Applications (Nova Publishers, NY, USA, 2008) and Software Security Engineering: Design and Applications (Nova Publishers, NY, USA, 2011). He is also an edited co-author of a book, Handbook of Research in Software Engineering (IGI, 2010) and has edited the book KE for SDLC (2011). He has widely authored published journal articles, book chapters and conferences materials on various advanced topics in software engineering and education. He received his Master's from the Indian Institute of Technology, Madras and from Madurai Kamaraj University, Madurai, India. He is a member of various professional organizations and computer societies: IEEE, ACM, Fellow of BCS, and Fellow of HEA. He was invited as a speaker to the 5th international symposium on SOA Cloud 2012, London. Muthu's research projects and publications can be accessed at http://www.leedsbeckett.ac.uk/staff/dr-muthu-ramachandran/.

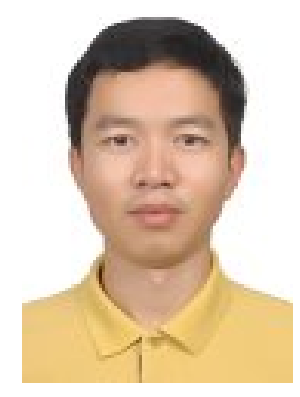

Jin Li is currently a professor and vice dean of School of Computer Science, Guangzhou University. He received his B.S. (2002) and M.S. (2004) from Southwest University and Sun Yat-sen University, both in Mathematics. He got his Ph.D degree in information security from Sun Yat-sen University at 2007. His research interests include design of secure protocols in Cloud Computing (secure cloud storage and outsourcing computation) and cryptographic protocols. He served as a senior research associate at Korea Advanced Institute of Technology (Korea) and Illinois Institute of Technology (U.S.A.) from 2008 to 2010, respectively. He has published more than 100 papers in international conferences and journals, including IEEE INFOCOM, IEEE TIFS, IEEE TPDS, IEEE TOC (16 papers in IEEE Transactions series journals) and ESORICS etc. His work has been cited more than 7620 times at Google Scholar and the $\mathrm{H}$-Index is 34 . He also served as program chairs and committee for many international conferences such as IEEE CSE 2017, IEEE EUC 2017, ISICA 2015 etc. He received three National Science Foundation of China (NSFC) Grants, including NSFC Outstanding Youth Foundation. He has been selected as one of science and technology new stars in Guangzhou and outstanding young scholar in Guangdong province. 


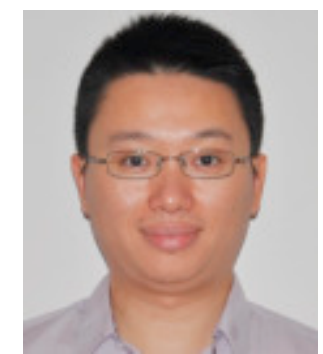

Dan Liao is a professor at University of Electronic Science and Technology of China (UESTC). His research interests are in the area of next generation network, wired and wireless computer communication networks and protocols. 